\title{
SOME DIOPHANTINE PROBLEMS ARISING FROM THE THEORY OF CYCLICALLY-PRESENTED GROUPS
}

\author{
R. W. K. ODONI \\ Department of Mathematics, University of Glasgow, Glasgow G12 8QW, UK
}

(Received 30 March, 1995; revised 26 August, 1998)

\begin{abstract}
Let $n \in \mathbb{N}$ and let $F_{n}$ be the free group on $n$ generators. Let $w$ be an arbitrary word in $F_{n}$, and let $\sigma$ be an $n$-cycle in $S_{n}$. We consider groups of the type $\Gamma(n, w)=F_{n} / N$, where $N$ is the normal closure in $F_{n}$ of the "cycled words" $w, \sigma(w)$, $\sigma^{2}(w), \ldots, \sigma^{n-1}(w)$, and solve, by means of classical algebraic number theory, the following problems.

A. When is $\Gamma(n, w)^{a b}$ infinite?

B. When is $\Gamma(n, w)$ a perfect group?
\end{abstract}

0. Introduction. Let $n \in \mathbb{N}$ and let $F_{n}$ be the free group on the $n$ symbols $Y_{1}, \ldots, Y_{n}$. For later purposes it is convenient to introduce extra "dummy symbols" $Y_{k}(k \in \mathbb{Z})$, such that $Y_{k}=Y_{l}$ wherever $k \equiv l(\bmod n)$. Now let $\sigma$ be a permutation of $\{1, \ldots, n\}$.

The map $Y_{i} \mapsto Y_{\sigma(i)}(1 \leq i \leq n)$ extends uniquely to an automorphism of $F_{n}$, which we shall also denote by $\sigma$, so that $\sigma\left(Y_{i}\right)=Y_{\sigma(i)}(1 \leq i \leq n)$.

Now let $w \in F_{n}$, and let $\sigma_{n}$ be the $n$-cycle $(12 \ldots n)$. Groups of the type

$$
\begin{aligned}
\Gamma(n, w) & =<Y_{1}, \ldots, Y_{n} \mid \sigma_{n}(w), \ldots, \sigma_{n}^{n}(w)> \\
& =F_{n} / N,
\end{aligned}
$$

where $N$ is the normal closure in $F_{n}$ of $\sigma_{n}(w), \ldots, \sigma_{n}^{n}(w)$, are called cyclically-presented, and have been studied by various authors-see e.g. $[\mathbf{2 , 4 , 6 , 7 , 1 0 , 1 1 ]}$. This paper addresses certain problems relating to the structure of the abelianization $\Gamma(n, w)^{a b}$ of the typical cyclically-presented $\Gamma(n, w)$. In particular we consider the following questions.

Problem A. When is $\Gamma(n, w)^{a b}$ infinite?

Problem B. When is $\Gamma(n, w)$ a perfect group; i.e. when is $\Gamma(n, w)^{a b}$ trivial?

There is a standard procedure (see e.g. $[\mathbf{7 , 8}]$ ) which reduces these problems to questions about ideals in the (commutative) group ring $\mathbb{Z} C_{n}$, where $C_{n}$ is cyclic of order $n$. We now briefly describe this.

For $g \in F_{n}$ let $\bar{g}$ be the image of $g$ under the natural epimorphism $F_{n} \rightarrow F_{n}^{a b}$. If $\bar{w}=\bar{Y}_{0}^{c_{c}} \ldots \bar{Y}_{n-1}^{c_{n-1}}$, with the $c_{i}$ in $\mathbb{Z}$, we introduce the polynomial $f(x)=f_{w}(x)=$ $\sum_{j<n} c_{j} x^{j} \in \mathbb{Z}[x]$.

The action of $C_{n}=<\sigma_{n}>\subseteq \operatorname{Aut}\left(F_{n}\right)$ on $F_{n}$ makes $F_{n}^{a b}$ into a left $\mathbb{Z} C_{n}$-module, and indeed $F_{n}^{a b} \cong \mathbb{Z} C_{n}$ as left $\mathbb{Z} C_{n}$-modules. Moreover $\Gamma(n, w)^{a b}$ is also a left $\mathbb{Z} C_{n^{-}}$ module, and we have an isomorphism 


$$
\Gamma(n, w)^{a b} \cong \mathbb{Z} C_{n} / f\left(\sigma_{n}\right) \mathbb{Z} C_{n}
$$

both as left $\mathbb{Z} C_{n}$-modules, and as $\mathbb{Z}$-modules (if we use additive notation for the group law in $\left.\Gamma(n, w)^{a b}\right)$.

As we show in $\S 1, \Gamma(n, w)^{a b}$ is infinite if and only if $f\left(\sigma_{n}\right)$ is a zero-divisor in $\mathbb{Z} C_{n}$, and $\Gamma(n, w)^{a b}$ is trivial if and only if $f\left(\sigma_{n}\right)$ is a unit in $\mathbb{Z} C_{n}$. (In slightly disguised notation, these results appear in $[\mathbf{7 , 8}]$.)

Our first main result concerns the case where $f(x) \in \mathbb{Z}[x]$ is fixed and $n$ varies in $\mathbb{N}$. Since, for each $n$, we may find (several) $w \in F_{n}$ yielding our given $f$ via the above procedure, the following theorem yields some useful information about Problems A and B.

TheOREM 1. Let $f(x) \in \mathbb{Z}[x], \operatorname{deg} f \geq 1$ with $f$ irreducible. For $n \in \mathbb{N}$ let $C_{n}=<\sigma_{n}>$ be a cyclic group of order $n$. Then

(i) $f\left(\sigma_{n}\right)$ is a zero-divisor in $\mathbb{Z} C_{n}$ if and only if $f(x)= \pm \Phi_{m}(x)$ for some $m \mid n$;

(ii) there are infinitely many $n \in \mathbb{N}$ such that $f\left(\sigma_{n}\right)$ is a unit in $\mathbb{Z} C_{n}$ if and only if $f(x)= \pm x$ or $\pm \Phi_{m}(x)$ for some $m>1$ not a prime-power. In the latter case, $f\left(\sigma_{n}\right)$ is a unit if and only if $m \mid \operatorname{gcd}(m, n)>1$ and is not a prime-power.

REMARKS. (i) In the above, for $m \in \mathbb{N}, \Phi_{m}(x)$ is the minimum polynomial for $\zeta_{m}=e^{2 \pi i / m}$ over $\mathbb{Q} ; \Phi_{m}(x)$ is monic in $\mathbb{Z}[x]$ of degree $\phi(m)$, where $\phi$ is Euler's totient function.

(ii) Since $\mathbb{Z}[x]$ is a unique factorisation domain, the results of Theorem 1 can be easily modified to cover the case where $f(x)$ is not irreducible. One simply notes that $f\left(\sigma_{n}\right)$ is a zero-divisor if and only if $g\left(\sigma_{n}\right)$ is a zero-divisor for some irreducible factor $g(x)$ of $f(x)$ in $\mathbb{Z}[x]$, while $f\left(\sigma_{n}\right)$ is a unit if and only if $g\left(\sigma_{n}\right)$ is a unit for every irreducible factor $g$ of $f$.

The remainder of the paper is devoted to the complete solution of Problems A and $\mathrm{B}$ for the case in which $f(x)=x^{t}-x+1$, where $t \geq 2$ and $n \geq 1$ are arbitrary. We prove the following result.

Theorem 2. For $t, n \in \mathbb{N}$, with $t \geq 2$,

(i) $\sigma_{n}^{t}-\sigma_{n}+1$ is a zero-divisor in $\mathbb{Z} C_{n}$ if and only if $n \equiv 0(\bmod 6)$ and $t \equiv 2(\bmod 6)$

(ii) for $\operatorname{gcd}(n, 6)=1, \sigma_{n}^{t}-\sigma_{n}+1$ is a unit in $\mathbb{Z} C_{n}$ if and only if $t \equiv 1$ or $2(\bmod n)$;

(iii) for $\operatorname{gcd}(n, 6)>1, \sigma_{n}^{t}-\sigma_{n}+1$ is a unit in $\mathbb{Z} C_{n}$ if and only if $t \equiv 1(\bmod n)$.

The principal ingredients in our proof of Theorem 2 are classical results on units in $\mathbb{Z}\left[\zeta_{m}\right]$, mostly due to Kronecker and Kummer.

I am indebted to Professor J. Howie (Heriot-Watt University) for drawing my attention to Problems A and B.

1. Preliminary results. We begin with some simple properties of $\mathbb{Q} C_{n}$ and $\mathbb{Z} C_{n}$ $(n \in \mathbb{N})$. We consider $\mathbb{Q} C_{n}$ as a $\mathbb{Q}$-algebra of dimension $n$.

For $\lambda \in \mathbb{Q} C_{n}$ let $L(\lambda)$ be the $\mathbb{Q}$-linear map $\alpha \mapsto \lambda \alpha$ on $\mathbb{Q} C_{n}$. The eigenvalues in $\mathbb{C}$ of $L\left(\sigma_{n}\right)$ are the $\theta$ with $\theta^{n}=1$, and for $g(x) \in \mathbb{Q}[x]$, the eigenvalues of $L\left(g\left(\sigma_{n}\right)\right)$ are the $g(\theta),\left(\theta^{n}=1\right)$, so that

$$
\operatorname{det} L\left(g\left(\sigma_{n}\right)\right)=\prod_{\theta^{n}=1} g(\theta) \in \mathbb{Q} \text {. }
$$


Now let $f(x) \in \mathbb{Z}[x]$. Then $f\left(\sigma_{n}\right)$ is a zero-divisor in $\mathbb{Z} C_{n}$ if and only if it is a zerodivisor in $\mathbb{Q} C_{n}$, if and only if $\operatorname{det} L\left(f\left(\sigma_{n}\right)\right)=0$, if and only if $\prod_{\theta^{n}=1} f(\theta)=0$.

Now suppose that $f(x) \in \mathbb{Z}[x]$ but $\operatorname{det} L\left(f\left(\sigma_{n}\right)\right) \neq 0$.

Let $M=L\left(f\left(\sigma_{n}\right)\right)$; it is a non-singular $\mathbb{Q}$-linear map on $\mathbb{Q} C_{n}$, while $M\left(\mathbb{Z} C_{n}\right)$ is a $\mathbb{Z}$-submodule of $\mathbb{Z} C_{n}$ of rank $n=\operatorname{rank} \mathbb{Z} C_{n}$. By "elementary divisor theory", $M\left(\mathbb{Z} C_{n}\right)$ has $\mathbb{Z}$-module index in $\mathbb{Z} C_{n}$ equal to $|\operatorname{det} M|$ or, equivalently,

$$
\mathbb{Z} C_{n} / f\left(\sigma_{n}\right) \mathbb{Z} C_{n}=|\operatorname{det} M|=\left|\prod_{\theta^{n}=1} f(\theta)\right|
$$

We also see from the above that, for $f \in \mathbb{Z}[x], \mathbb{Z} C_{n} / f\left(\sigma_{n}\right) \mathbb{Z} C_{n}$ is infinite if and only if $\prod_{\theta^{n}=1} f(\theta)=0$, if and only if $f\left(\sigma_{n}\right)$ is a zero-divisor in $\mathbb{Z} C_{n}$. Also, by (1.1), $f\left(\sigma_{n}\right)$ is a unit in $\mathbb{Z} C_{n}$ if and only if $\prod_{\theta^{n}=1} f(\theta)= \pm 1$.

To summarise, we put

$$
R_{n}(f)=\prod_{\theta^{n}=1} f(\theta) \in \mathbb{Z} \quad(f(x) \in \mathbb{Z}[x]) .
$$

Then we have proved the following result.

Lemma 1.1. $\mathbb{Z} C_{n} / f\left(\sigma_{n}\right) \mathbb{Z} C_{n}$ is infinite if and only if $R_{n}(f)=0$, and has order 1 if and only if $R_{n}(f)= \pm 1$.

We now turn to standard classical results from algebraic number theory needed for the proofs of Theorems 1 and 2. Reference [9] is a convenient source for most of these.

Lemma 1.2. (Kronecker). Let $\beta=\beta_{1}$ be an algebraic integer, and let $\beta_{1}, \ldots, \beta_{k}$ be the conjugates of $\beta$ over $\mathbb{Q}$. Suppose that $\max \left|\beta_{j}\right| \leq 1$. Then either $\beta_{1}=\ldots=\beta_{k}=0$ (and then $k=1$ ), or $\beta$ is a root of unity. ${ }^{j}$

For a proof see $[9$, p.46]

Lemma 1.3. Let $m \in \mathbb{N}, K=\mathbb{Q}\left(\zeta_{m}\right)$, where $\zeta_{m}=e^{2 \pi i / m}$. The roots of unity in $K$ are precisely the $\pm \zeta_{m}^{k}(k \in \mathbb{Z})$.

For a proof see $[9$, p. 170]

Lemma 1.4. Let $t \in \mathbb{N}, t \geq 2$, and let $f(x)=x^{t}-x+1 \in \mathbb{Z}[x]$. Then $f$ has a (complex) zero $\lambda$ of absolute value 1 if and only if $t \equiv 2(\bmod 6)$, in which case $\lambda= \pm \zeta_{6}$.

Proof. Suppose that $f(\lambda)=0$, where $\lambda \in \mathbb{C}$ has $|\lambda|=1$. Then $f(\bar{\lambda})=0$ while $\bar{\lambda}=\lambda^{-1}$. Hence $\lambda^{t}=\lambda-1$ and $\lambda^{-t}=\lambda^{-1}-1$, so that $1=\lambda^{t} \lambda^{-t}=(\lambda-1)\left(\lambda^{-1}-1\right)=$ $2-\lambda-\lambda^{-1}$, and so $\lambda^{2}-\lambda+1=0$. Thus $\lambda= \pm \zeta_{6}$ while $0 \neq \lambda^{t}=\lambda-1=\lambda^{2}$. Hence $\lambda^{t-2}=1$. Since $\pm \zeta_{6}$ has order 6 in $\mathbb{C}^{*}$ we see that $t \equiv 2(\bmod 6)$.

Conversely if $t \equiv 2(\bmod 6)$, then $\lambda= \pm \zeta_{6}$ satisfies $\lambda^{t}=\lambda^{2}$ and $\lambda^{t}-\lambda+1=$ $\lambda^{2}-\lambda+1=0$, so that $f(x)=x^{t}-x+1$ has $f(\lambda)=0$ and $|\lambda|=1$. 
2. Proof of Theorem 1. We begin with an elementary calculation of $R_{n}(f)$.

LEMMA 2.1. Let $f(x) \in \mathbb{Z}[x], \operatorname{deg} f=k \geq 1$, and suppose that $f(x)=c \prod_{j \leq k}\left(x-\beta_{j}\right)$ in $\mathbb{C}[x]$, where $0 \neq c \in \mathbb{Z}$. Then $R_{n}(f)$ of (1.2) equals $\left((-1)^{k} c\right)^{n} \prod_{j \leq k}\left(\beta_{j}^{n}-1\right)$.

Proof.

$$
\begin{aligned}
R_{n}(f) & =\prod_{\theta^{n}=1}\left\{c \prod_{j \leq k}\left(\theta-\beta_{j}\right)\right\} \\
& =c^{n} \prod_{\theta} \prod_{j}\left(\theta-\beta_{j}\right) \\
& =c^{n}(-1)^{n k} \prod_{j} \prod_{\theta}\left(\beta_{j}-\theta\right) \\
& =c^{n}(-1)^{n k} \prod_{j \leq k}\left(\beta_{j}^{n}-1\right) .
\end{aligned}
$$

Now suppose that $f(x)$ is irreducible in $\mathbb{Z}[x]$, of degree $k \geq 1$, and that $R_{n}(f)= \pm 1$, for all $n \in N_{1}$, an infinite subset of $\mathbb{N}$.

If $k=1$ then it is clear from Lemma 2.1 that $c= \pm 1$ and that $f(x)= \pm\left(x-\beta_{1}\right)$ with $\beta \in \mathbb{Z}$, so that $\beta_{1}^{n}-1= \pm 1$, for all $n \in N_{1}$; i.e. $\beta_{1}^{n}=0$ or 2 , for all $n \in N_{1}$. If $\beta_{1} \neq 0$, then $\beta_{1}^{n}=2$, for infinitely many $n$, which is impossible since $\beta_{1} \in \mathbb{Z}$. Thus $\beta_{1}=0$, and so $f(x)= \pm x$ and $R_{n}(f)= \pm 1$, for all $n \in \mathbb{N}$.

We may now assume that $k \geq 2$. Put $a=|c| \geq 1$. Then there is an infinite subset $N_{2}$ of $N_{1}$ such that

$$
a^{n} \prod_{j \leq k}\left(\beta_{j}^{n}-1\right)=\zeta \quad\left(\forall n \in N_{2}\right),
$$

where $\zeta$ is some fixed choice of \pm 1 . We partition $\{1, \ldots k\}$ into three parts (some of them possible empty); thus let

$$
A=\left\{j ;\left|\beta_{j}\right|<1\right\}, \beta=\left\{j ;\left|\beta_{j}\right|=1\right\}, C=\left\{j ;\left|\beta_{j}\right|>1\right\} .
$$

We put $h=\prod_{j \in C}\left|\beta_{j}\right|$, with the convention that empty products equal 1 . We shall first show that $C=\emptyset$. If this is false, then $h>1$ and so $a h>1$. We shall rule out the latter case.

Suppose, aiming for a contradiction, that $a h>1$, given (2.1). Letting $n \rightarrow \infty$ through $\mathrm{N}_{2}$ we have

$$
\prod_{j \in A}\left(\beta_{j}^{n}-1\right) \sim(-1)^{A} \text { while } \prod_{j \in C}\left|\beta_{j}^{n}-1\right| \sim h^{n} .
$$

Applying (2.1), we have

$$
\prod_{j \in B}\left|\beta_{j}^{n}-1\right| \sim(a h)^{-n}
$$


as $n \rightarrow \infty$ through $N_{2}$. As $a h>1$ we immediately see that $B \neq \emptyset$. Then there is some $d>0$ in $\mathbb{R}$ and an infinite subset $N_{3}$ of $N_{2}$ such that, for some $r \in B$, we have

$$
\left|\beta_{r}^{n}-1\right| \leq e^{-n d} \quad\left(\forall n \in N_{3}\right) .
$$

By Gel'fond's theorem [5, p. 28], (2.3) is impossible unless $\beta_{r}$ is a root of unity. (Recall that the $\beta_{j}$ are algebraic numbers.) Let $\beta_{r}$ be a primitive root of unity of order $m \in \mathbb{N}$. Then $\Phi_{m}(x) \mid f(x)$ in $\mathbb{Z}[x]$. As both are irreducible, we have $f(x)= \pm \Phi_{m}(x)$. Hence $a=|c|=1$ and $\left|\beta_{j}\right|=1$ for all $j \leq k=\phi(m)$. This forces $C=\emptyset$ and $h=1$, so that $a h=1$, a contradiction.

It follows that $a h \leq 1$ in (2.1). Since $a=|c| \geq 1$ we have $h \leq 1$. Hence (2.1) implies that $a=1, h=1$ and $C=\emptyset$, so that $\beta_{1}, \ldots, \beta_{k}$ are algebraic integers with $\max _{j \leq k}\left|\beta_{j}\right| \leq 1$, while the $\beta_{j}$ are the conjugates of $\beta_{1}$. Since $k \geq 2$ this forces $f(x)= \pm \Phi_{m}(x)$ with $\phi(m)=k \geq 2$ and so $m \geq 3$.

If $m \geq 3$ is a prime-power, then $\zeta_{m}-1$ generates a maximal ideal $\mathbf{P}$ in $\mathbb{Z}\left[\zeta_{m}\right]$, and then Lemma 2.1 shows that $R_{n}(f) \in \mathbf{P}$, for all $n \in \mathbb{N}$, a contradiction.

Finally suppose that $m \geq 3$ is not a prime-power. Then $\zeta_{m}-1$ is a unit in $\mathbb{Z}\left[\zeta_{m}\right]$, since $\Phi_{m}(1)=1$, while

$$
R_{n}(f)= \pm \prod_{r \in V}\left(\zeta_{m}^{r n}-1\right)
$$

where $V=\{r \in \mathbb{Z} ; 0<r<m, \operatorname{gcd}(r, m)=1\}$. But, for $r \in V, \zeta_{m}^{r n}$ is a primitive root of unity of order $m^{*}=m / \operatorname{gcd}(m, n)$, so that $\zeta_{m}^{r m}-1$ is a non-unit in $\mathbb{Z}\left[\zeta_{m^{*}}\right]$ unless $m^{*}>1$ is not a prime power. If the latter fails to hold, then $R_{n}(f)$ is a non-unit in $\mathbb{Z}\left[\zeta_{m^{*}}\right]$ and so cannot be \pm 1 . To complete the proof of Theorem 1 we have

$$
R_{n}(f)= \pm N_{K / \mathbb{Q}}\left(\zeta_{m}^{n}-1\right)
$$

where $N_{K / \mathbb{Q}}$ is the norm from $K=\mathbb{Q}\left(\zeta_{m}\right)$ to $\mathbb{Q}$, and so $R_{n}(f)= \pm\left\{N_{L / \mathbb{Q}}\left(\zeta_{m^{*}}-1\right)\right\}^{g}$, where $g \in \mathbb{N}$ and $L=\mathbb{Q}\left(\zeta_{m^{*}}\right)$.

(Here, as before, $m^{*}=m / \operatorname{gcd}(m, n)$.)

In particular $R_{n}(f)= \pm 1$ if and only if $\zeta_{m^{*}}-1$ is a unit in $\mathbb{Z}\left[\zeta_{m^{*}}\right]$, and this certainly holds if $m^{*}>1$ is not a prime-power.

3. Proof of Theorem 2. Let $t \in \mathbb{N}, t \geq 2$. Throughout this section $f(x)$ will be $x^{t}-x+1 \in \mathbb{Z}[x]$.

We first dispose of the question of when $R_{n}(f)= \pm 1$; i.e. when $f\left(\sigma_{n}\right)$ is a unit in $\mathbb{Z} C_{n}$. The condition $R_{n}(f)= \pm 1$ is clearly equivalent to

$$
f\left(\zeta_{d}\right) \text { is a unit in } \mathbb{Z}\left[\zeta_{d}\right],(\forall d \mid n),
$$

and this formulation turns out to be very fruitful.

Lemma 3.1. Let $n \in \mathbb{N}, \operatorname{gcd}(n, 6)=1$. Then $f\left(\zeta_{n}\right)$ is a unit in $\mathbb{Z}\left[\zeta_{n}\right]$ if and only if $t \equiv 1$ or $2(\bmod n)$. 
Proof. The "if" part is easy. For $t \equiv 1(\bmod n)$ we have $f\left(\zeta_{n}\right)=\zeta_{n}^{t}-\zeta_{n}+1=1$, while if $t \equiv 2(\bmod n)$ then $f\left(\zeta_{n}\right)=\zeta_{n}^{2}-\zeta_{n}+1$ and moreover $f(\theta)=\theta^{2}-\theta+1$ whenever $\theta^{n}=1$, so that by Lemma 2.1 we have

$$
R_{n}(f)= \pm \prod_{\lambda^{2}=\lambda-1}\left(\lambda^{n}-1\right)= \pm 1(\text { since } \operatorname{gcd}(n, 6)=1)
$$

In particular $f\left(\zeta_{n}\right)$ is a unit in $\mathbb{Z}\left[\zeta_{n}\right]$ if $t \equiv 1$ or $\left.2(\bmod n)\right)$.

Suppose, conversely, that $\operatorname{gcd}(n, 6)=1$, and that $f\left(\zeta_{n}\right)$ is a unit. The case $n=1$ is trivial (since $f(1)=1$ is a unit for any $t \geq 2$ ).

We may now suppose that $n \geq 5$. Let $\lambda=f\left(\zeta_{n}\right)$ be a unit in $\mathbb{Z}\left[\zeta_{n}\right]$. Then so is $\lambda^{\sigma}$ for all $\sigma \in G:=\operatorname{Gal}\left(\mathbb{Q}\left(\zeta_{n}\right) / \mathbb{Q}\right)$. Let $\tau$ be complex-conjugation in $G$.

Since $G$ is abelian, we have

$$
\left|\mu^{\sigma}\right|^{2}=\mu^{\sigma} \mu^{\sigma \tau}=\left(\mu \mu^{\tau}\right)^{\sigma}=1,
$$

for all $\sigma \in G$, where $\mu=\lambda^{\tau} \lambda^{-1}$ is a unit in $\mathbb{Z}\left[\sigma_{n}\right]$.

By Lemma 1.2, $\mu$ is a root of unity in $\mathbb{Q}\left(\zeta_{n}\right)$, and thus has the form $\pm \zeta_{n}^{k}(k \in \mathbb{Z})$.

Since $\mu=\lambda^{\tau} \lambda^{-1}$ and $\lambda=f\left(\zeta_{n}\right)$, we have

$$
\zeta^{-t}-\zeta^{-1}+1=s \zeta^{k}\left(\zeta^{t}-\zeta+1\right)
$$

where $\zeta=\zeta_{n}$ and $s= \pm 1$.

Case 1: $s=-1$. We shall rule this out, by the following argument. By (3.2) we have that

$$
w_{1}+w_{2}+w_{3}+w_{4}=z_{1}+z_{2}+z_{3}+z_{4},
$$

where $w_{1}=\zeta^{-t}, w_{2}=1, w_{3}=\zeta^{k+t}, w_{t}=\zeta^{k}, z_{1}=\zeta^{-1}, z_{2}=\zeta^{k+1}$, and $z_{3}=z_{4}=0$.

Applying to (3.3) the elements $\zeta \mapsto \zeta^{r}$ of $G=\mathrm{Gal} \mathbb{Q}(\zeta) / \mathbb{Q}$ for $r=1,2,3,4$ (recalling that $\operatorname{gcd}(n, 6)=1)$, we see that

$$
\sum_{j \leq 4} w_{j}^{r}=\sum_{j \leq 4} z_{j}^{r} \quad(1 \leq r \leq 4) .
$$

The classical Newton-Waring identities connecting symmetric power-sums and elementary symmetric functions yield from (3.4) that the sets $\left\{w_{1}, \ldots, w_{4}\right\}$ and $\left\{z_{1}, \ldots, z_{4}\right\}$ coincide. However $0 \in\left\{z_{1}, \ldots, z_{4}\right\}$ but $0 \notin\left\{w_{1}, \ldots, w_{4}\right\}$, a contradiction. Hence the case $s=-1$ cannot occur. We are left with Case 2.

Case 2: $s=1$. Then we have

$$
w_{1}+w_{2}+w_{3}=z_{1}+z_{2}+z_{3}
$$

where $w_{1}=\zeta^{-t}, w_{2}=1, w_{3}=\zeta^{k+1}, z_{1}=\zeta^{-1}, z_{2}=\zeta^{k+2}, z_{3}=\zeta^{k}$.

This time we apply to (3.5) the elements $\zeta \mapsto \zeta^{r}$ of $G(r=1,2,3)$ and find that

$$
\left\{\zeta^{-t}, 1, \zeta^{k+1}\right\}=\left\{\zeta^{-1}, \zeta^{k+t}, \zeta^{k}\right\}
$$


In particular $1=\zeta^{k+t}$ or $\zeta^{k}$, the case $\zeta^{-1}=1$ being ruled out since $\zeta=\zeta^{n}$ and $n \geq 5$. If $1=\zeta^{k+t}$, then $\left\{\zeta^{-t}, \zeta^{k+1}\right\}=\left\{\zeta^{-1}, \zeta^{k}\right\}$ so that $\zeta^{k+2}=1=\zeta^{k+t}, \zeta^{t-2}=1$ and $t \equiv 2(\bmod n)$. If $1=\zeta^{k}$, then $\left\{\zeta^{-t}, \zeta\right\}=\left\{\zeta^{-1}, \zeta^{t}\right\}$, and so $\zeta^{t} \equiv \zeta^{-t}$ or $\zeta$.

If $\zeta^{t}=\zeta^{-t}$, then $\zeta_{n}^{2 t}=1$, and, as $n$ is odd, $\zeta^{t}=1$, in which case $\{1, \zeta\}=\left\{\zeta^{-1}, 1\right\}$, clearly false. Hence we have $\zeta^{t}=\zeta$ and thus $t \equiv 1(\bmod n)$. This proves the lemma.

Before we proceed further we note a further property of $R_{n}(g)$ for $n \in \mathbb{N}$, $g \in \mathbb{Z}[x]$. It is clear from (1.2) that

$$
R_{n}(g) \in R_{d}(g) \mathbb{Z}\left[\zeta_{n}\right]
$$

whenever $d \mid n$. In particular if $R_{n}(g) \neq 0$, the $R_{d}(g) \neq 0$ and we have that $R_{n}(g) / R_{d}(g) \in \mathbb{Q}_{\cap} \mathbb{Z}\left[\zeta_{n}\right]=\mathbb{Z}$, so that $R_{d}(g)$ divides $R_{n}(g)$ in $\mathbb{Z}$.

Lemma 3.2. Let $p=2$ or 3 and let $n$ be a power of $p$. Then $R_{n}(f)= \pm 1$ if and only if $t \equiv 1(\bmod n)$.

Proof. (i) If $t \equiv 1(\bmod n)$ we have $\theta^{t}-\theta+1=1$ whenever $\theta^{n}=1$ and so $R_{n}(f)= \pm 1$.

(ii) We now prove by induction on $k \geq 0$ that if $n=p^{k}$ and $R_{n}(f)= \pm 1$ then $t \equiv 1(\bmod n)$. For $k=0$ this is vacuously true. For $k=1$ we have $R_{p}(f)=\prod_{\theta^{p}=1} f(\theta)$. If $p=2$ we have $R_{p}(f)=R_{2}(f)=f(1) f(-1)=f(-1)=2+(-1)^{t}= \pm 1$ if and only if $t \equiv 1(\bmod 2)$; i.e. $t \equiv 1(\bmod n)$ as $n=2$ here.

If $p=3$ we have $R_{p}(f)=R_{3}(f)=f(1) f\left(\zeta_{3}\right) f\left(\zeta_{3}^{2}\right)=f\left(\zeta_{3}\right) f\left(\bar{\zeta}_{3}\right)=\left|f\left(\zeta_{3}\right)\right|^{2} \geq 0$ and $R_{3}(f)= \pm 1$ if and only if $\zeta_{3}^{t}-\zeta_{3}+1$ is a unit in $\mathbb{Z}\left[\zeta_{3}\right]$. This happens if and only if $t \equiv 1(\bmod 3)$, since the units in $\mathbb{Z}\left[\zeta_{3}\right]$ are the powers of $\zeta_{6}$.

This covers the case $k=1$. Now suppose that $k>1$ and that $R_{p^{s}}(f)= \pm 1$ if and only if $t \equiv 1\left(\bmod p^{s}\right)$ holds whenever $0 \leq s \leq k$.

Suppose that $R_{p^{k+1}}(f) \pm 1$. Then by (3.1), we have $R_{p^{k}}(f)= \pm 1$, so that $t \equiv 1\left(\bmod p^{k}\right)$ and $t \equiv 1+c p^{k}\left(\bmod p^{k+1}\right)$, for some $c \in \mathbb{Z}$. We must show that $c \in p \mathbb{Z}$. We put $\zeta=\zeta_{p^{k+1}}$ and $\omega=\zeta_{p}$, and let $N(\ldots)$ be the norm map from $\mathbb{Q}(\zeta)$ to $\mathbb{Q}(\omega)$.

We have $f(\zeta)=\zeta^{1+c p^{k}}-\zeta+1=\zeta\left(w^{c}-1\right)+1$, and, as $R_{p^{k+1}}(f)= \pm 1, f(\zeta)$ is a unit in $\mathbb{Z}[\zeta]$.

Since the characteristic polynomial for $\zeta$ over $\mathbb{Q}(\omega)$ is $X^{p^{k}}-\omega$ we see that $N(f(\zeta))=1-\omega\left(1-\omega^{c}\right)^{p^{k}}$ is a unit in $\mathbb{Z}[\omega]$. As $p=2$ or $3, \mathbb{Q}(\omega)$ is $\mathbb{Q}$ or an imaginary quadratic field, and so Lemma 1.3 implies that

$$
1-\omega\left(1-\omega^{c}\right)^{p^{k}}=s \omega^{m}(s= \pm 1, m \in \mathbb{Z}) .
$$

If $p=2,(3.7)$ gives

$$
1+\left(1-(-1)^{c}\right)^{2^{k}}=s(-1)^{m}= \pm 1
$$

and if $c$ were odd, we would have $1+2^{2^{k}}= \pm 1$, which is impossible, so that $c$ is even and $t \equiv 1\left(\bmod 2^{k+1}\right)$, as required., If $p=3$, (3.8) gives

$$
1-s \zeta_{3}^{m}=\zeta_{3}\left(1-\zeta_{3}^{c}\right)^{3^{k}}
$$

If $c \notin 3 \mathbb{Z}$, then $\pi \| 1-\zeta_{3}^{c}$ in $\mathbb{Z}\left[\zeta_{3}\right]$, where $\pi$ is the prime $1-\zeta_{3}$, so that $\pi^{3^{k}} \|$ (righthand side of (3.9)) But the left-hand side of (3.9) is one of $1 \pm 1,1 \pm \zeta_{3}$ or $1 \pm \zeta_{3}^{2}$, none of which is exactly divisible by $\pi^{3^{k}}$ (since $k \geq 1$ ). Hence $c \in 3 \mathbb{Z}$ and so $t \equiv 1\left(\bmod 3^{k+1}\right)$, as required. 
Lemma 3.3. Let $p \geq 5$ be prime. Then

$$
R_{2_{p}}(f)= \pm 1 \text { if and only if } t \equiv 1(\bmod 2 p)
$$

and

$$
R_{3_{p}}(f)= \pm 1 \text { if and only if } t \equiv 1(\bmod 3 p) .
$$

Proof. Let $q=2$ or 3 . If $R_{p q}(f)= \pm 1$, then $R_{q}(f)= \pm 1$, so that $t \equiv 1(\bmod q)$. Also $R_{p}(f)= \pm 1$, so that $t \equiv 1$ or $2(\bmod p)$. If $t \equiv 1(2 \bmod p)$, then we have $t \equiv 1(\bmod p q)$, as required, and, conversely, if $t \equiv 1(\bmod p q)$, then $f(\theta)=1$ whenever $\theta^{p q}=1$, so that $R_{p q}(f)= \pm 1$. It remains to eliminate the possibility that $t \equiv 1(\bmod q)$ and $t \equiv 2(\bmod p)$. Suppose that these congruences hold, and the $R_{p q}(f)= \pm 1$; then $f(\theta)$ must be a unit in $\mathbb{Z}\left[\zeta_{p q}\right]$ whenever $\theta^{p q}=1$. In particular, for every $b \in \mathbb{Z}, f\left(\zeta_{p} \zeta_{q}^{b}\right)=\left(\zeta_{p}^{2}-\zeta_{p}\right) \zeta_{q}^{b}+1$ must be a unit, and hence so is $\zeta_{p}^{2}-\zeta_{p}+\zeta_{q}^{-b}$.

Case $q=2$. We see that $\zeta_{p}^{2}-\zeta_{p}-1$ must be a unit in $\mathbb{Z}\left[\zeta_{p}\right]$. Let $g(X)=X^{2}-X-1$. Then $g(1)=-1$ and $g\left(\zeta_{p}\right)$ is a unit in $\mathbb{Z}\left[\zeta_{p}\right]$; hence so is $g\left(\zeta_{p}^{\sigma}\right)$, for all $\sigma \in \operatorname{Gal}\left(\mathbb{Q}\left(\zeta_{p}\right) / \mathbb{Q}\right)$. In particular, by Lemma 2.1,

$$
\prod_{\theta^{p}=1} g(\theta)= \pm 1= \pm\left(\lambda_{1}^{p}-1\right)\left(\lambda_{2}^{p}-1\right)
$$

where $\lambda_{1}>\lambda_{2}$ are the zeros $\frac{1}{2}(1 \pm \sqrt{5})$ of $g$.

Now $p \geq 5$ is odd and $\lambda_{2}=-\lambda_{1}^{-1}$, so that $\left(\lambda_{1}^{p}-1\right)\left(\lambda_{2}^{p}-1\right)$ must be 1 , by (3.10).

But $\lambda_{1}>\frac{3}{2}$ and so

$$
1=\left(\lambda_{1}^{p}-1\right)\left(\lambda_{2}^{p}-1\right)=\left(\lambda_{1}^{p}-1\left(1+\lambda_{1}^{-p}\right)>\left(\frac{3}{2}\right)^{5}-1,\right.
$$

a contradiction. Hence if $q=2$ we must have $t \equiv 1(\bmod p q)$ if $R_{p q}(f)= \pm 1$, as required.

Case $q=3$. This time we have $\zeta_{p}^{2}-\zeta_{p}+\zeta_{3}^{-b}$ is a unit, for all $b \in \mathbb{Z}$. Taking $b=0,1,2$ and multiplying these units together we see that $1+\left(\zeta_{p}^{2}-\zeta_{p}\right)^{3}$ must be a unit in $\mathbb{Z}\left[\zeta_{p}\right]$. Let $\lambda=1-\zeta_{p}$. Then $\lambda \mathbb{Z}\left[\zeta_{p}\right]$ is a maximal ideal $\mathbf{P}$ in $\mathbb{Z}\left[\zeta_{p}\right]$, and $\mathbf{P}^{p-1}=p \mathbb{Z}\left[\zeta_{p}\right]$, while $N(\mathbf{P})=\# \mathbb{Z}\left[\zeta_{p}\right] / \mathbf{P}=p$.

Now, by hypothesis $\delta=1+\left(\zeta_{p}^{2}-\zeta_{p}\right)^{3}$ is a unit in $\mathbb{Z}\left[\zeta_{p}\right]$, while

$$
\delta \equiv 1-\lambda^{3}\left(\bmod \mathbf{P}^{4}\right)
$$

Let $\tau \in \operatorname{Gal}\left(\mathbb{Q}\left(\zeta_{p}\right) / \mathbb{Q}\right)$ be complex-conjugation. Then $\mathbf{P}^{\tau}=\mathbf{P}$ and $\lambda^{\tau}=1-\zeta_{p}^{-1}=$ $-\zeta_{p}^{-1} \lambda$ so that

$$
\delta^{\tau} \equiv\left(1+\lambda^{3}\right)\left(\bmod \mathbf{P}^{4}\right)
$$

However $\delta^{\tau}=s \zeta_{p}^{k} \delta(s= \pm 1, k \in \mathbb{Z})$, by Lemmas 1.2 and 1.3 , so that $s \zeta_{p}^{k}\left(1-\lambda^{3}\right) \equiv$ $1+\lambda^{3}\left(\bmod \mathbf{P}^{4}\right)$ and hence $s \zeta_{p}^{k}-1 \in \mathbf{P}^{3}$. Since $\zeta_{p} \equiv 1(\bmod \mathbf{P})$ we have $s \equiv 1(\bmod \mathbf{P})$. As $2 \notin \mathbf{P}$ we have $s=1$, and so $\delta^{\tau}=\zeta_{p}^{k} \delta$ and $\zeta_{p}^{k}-1 \in \mathbf{P}^{3}$. If $k \notin p \mathbb{Z}$ we have $\mathbf{P} \| \zeta_{p}^{k}-1$ and so $\delta^{\tau}=\delta$. But $\delta^{\tau} \equiv 1+\lambda^{3}\left(\bmod \mathbf{P}^{4}\right)$ by (3.12) and (3.13). From $\delta^{\tau}=\delta$ we see that $2 \in \mathbf{P}$, a contradiction. 
Thus there is no unit $\delta$ satisfying (3.12) and, in particular $1+\left(\zeta_{p}^{2}-\zeta_{p}\right)^{3}$ cannot be a unit in $\mathbb{Z}\left[\delta_{p}\right]$. Hence $R_{3 p}(f)$ cannot be \pm 1 unless $t \equiv 1(\bmod 3 p)$, as required.

We can now complete the proof of Theorem 2 .

Let $n \in \mathbb{N}$. If $n=1$, we have $R_{n}(f)=R_{1}(f)=1$, for all $t \geq 2$, and there is nothing more to prove. Now write $n=a b$, where $a=2^{r} 3^{s}(r, s \geq 0)$ and $\operatorname{gcd}(6, b)=1$. We may assume that $n=a b>1$.

If $a=1$ we use Lemma 3.1. If $b=1$ and $a>1$ we have from $R_{n}(f)= \pm 1$ that $R_{2^{r}}(f)= \pm 1$, so that $t \equiv 1\left(\bmod 2^{r}\right)$, and also $R_{3^{s}}(f)= \pm 1$, so that $t \equiv 1\left(\bmod 3^{s}\right)$. Hence $t \equiv 1(\bmod a)$; i.e. $t \equiv 1(\bmod n)$.

Finally, suppose that $a, b>1$. From $R_{n}(f)= \pm 1$, we have $R_{a}(f)= \pm 1$, so that $t \equiv(\bmod a)$, by the above. Also $R_{b}(f)$ must be \pm 1 , so that $t \equiv 1$ or $2(\bmod b)$.

We rule out the case $t \equiv 2(\bmod b)$ as follows. Since $a>1$ and $b>1, n$ has a divisor of the type $p q$, where $q=2$ or 3 and $p \geq 5$ is a prime divisor of $b$.

We must have $R_{p q}(f)= \pm 1$; hence $t \equiv 1(\bmod p)$, by Lemma 3.3 . Certainly $t \neq 2(\bmod b)$.

Since for every $n \in \mathbb{N}$ we certainly have $R_{n}(f)= \pm 1$ whenever $t \equiv 1(\bmod n)$, the proof of Theorem 2 is completed.

4. Concluding remarks. (a) In place of the Gel'fond-Baker results, one may use "Skolem's $p$-adic method" $[3$, p. 67, 228] to obtain Theorem 1. For general $f$ the latter approach has various advantages, since explicit $p$-adic bounds for the $n$ with $R_{n}(f)= \pm 1$ can be obtained from Strassmann's theorem [3, p. 62].

(b) The polynomial $f(X)=X^{t}-X+1$ was chosen in Theorem 2 since the corresponding groups $\Gamma(n, w)$ have attracted a good deal of attention (see the references in $\S 0$ ). However it is clear that the methods used in proving Theorem 2 will give useful information for more general $f$, particularly if $f$ has small height. (If $f(X)=\Sigma c_{j} X^{j}$, the height of $f$ is $\operatorname{deg}(f)+\Sigma\left|c_{j}\right|$.)

\section{REFERENCES}

1. A. Baker, Transcendental number theory (Cambridge University Press, 1975).

2. C. M. Campbell and E. T. Robertson, The order of certain metacyclic groups. Bull. London Math. Soc. 6 (1974), 312-314.

3. J. W. S. Cassels, Local fields, London Math. Soc. Student Text No. 3 (Cambridge University Press, 1986).

4. J. H. Conway, Solution to Advanced Problem 5327, American Math. Monthly 74 (1967), 91-93.

5. A. O. Gel'fond, Transcendental and algebraic numbers (Dover, New York, 1960).

6. D. L. Johnson, A note on the Fibonacci groups, Israel J. Math. 17 (1974), 277-282.

7. D. L. Johnson, Presentations of groups London Math. Soc. Student Text No. 15 (Cambridge University Press, 1990).

8. D. L. Johnson and R. W. K. Odoni, Some results on symmetrically-presented groups, Proc. Edinburgh Math. Soc. 37 (1994), 227-237.

9. W. Narkiewicz, Elementary and analytic theory of algebraic numbers (2nd ed., Springer Verlag, 1990).

10. R. M. Thomas, The Fibonacci groups revisited, in Groups, St. Andrews, 1989 (Cambridge University Press, 1994), 445-454.

11. R. M. Thomas, On a question of Kim concerning certain group presentations, Bull. Korean Math. Soc. 28 (1991), 219-224. 